\title{
A NEW APPROACH TO THE FUNDAMENTAL THEOREM OF SURFACE THEORY
}

\author{
PHILIPPE G. CIARLET, LILIANA GRATIE, AND CRISTINEL MARDARE
}

ABSTRACT. The fundamental theorem of surface theory classically asserts that, if a field of positive-definite symmetric matrices $\left(a_{\alpha \beta}\right)$ of order two and a field of symmetric matrices $\left(b_{\alpha \beta}\right)$ of order two together satisfy the Gauss and Codazzi-Mainardi equations in a connected and simply-connected open subset $\omega$ of $\mathbb{R}^{2}$, then there exists an immersion $\boldsymbol{\theta}: \omega \rightarrow \mathbb{R}^{3}$ such that these fields are the first and second fundamental forms of the surface $\boldsymbol{\theta}(\omega)$ and this surface is unique up to proper isometries in $\mathbb{R}^{3}$.

In this paper, we identify new compatibility conditions, expressed again in terms of the functions $a_{\alpha \beta}$ and $b_{\alpha \beta}$, that likewise lead to a similar existence and uniqueness theorem. These conditions take the form

$$
\partial_{1} \mathbf{A}_{2}-\partial_{2} \mathbf{A}_{1}+\mathbf{A}_{1} \mathbf{A}_{2}-\mathbf{A}_{2} \mathbf{A}_{1}=\mathbf{0} \text { in } \omega,
$$

where $\mathbf{A}_{1}$ and $\mathbf{A}_{2}$ are antisymmetric matrix fields of order three that are functions of the fields $\left(a_{\alpha \beta}\right)$ and $\left(b_{\alpha \beta}\right)$, the field $\left(a_{\alpha \beta}\right)$ appearing in particular through its square root. The unknown immersion $\boldsymbol{\theta}: \omega \rightarrow \mathbb{R}^{3}$ is found in the present approach in function spaces "with little regularity", viz., $W_{\mathrm{loc}}^{2, p}\left(\omega ; \mathbb{R}^{3}\right), p>2$.

\section{Une nouvelle approche du théorème fondamental de la théorie des surfaces.}

RÉSUMÉ. Le théorème fondamental de la théorie des surfaces affirme classiquement que, si un champ de matrices $\left(a_{\alpha \beta}\right)$ symétriques définies positives d'ordre deux et un champ de matrices $\left(b_{\alpha \beta}\right)$ symétriques d'ordre deux satisfont ensemble les équations de Gauss et Codazzi-Mainardi dans un ouvert $\omega \subset \mathbb{R}^{2}$ connexe et simplement connexe, alors il existe une immersion $\theta: \omega \rightarrow \mathbb{R}^{3}$ telle que ces deux champs soient les première et deuxième formes fondamentales de la surface $\boldsymbol{\theta}(\boldsymbol{\omega})$, et cette surface est unique aux isométries propres de $\mathbb{R}^{3}$ près.

Dans cet article, nous identifions de nouvelles conditions de compatibilité, exprimées à nouveau à l'aide des fonctions $a_{\alpha \beta}$ et $b_{\alpha \beta}$, qui conduisent aussi à un théorème analogue d'existence et d'unicité. Ces conditions sont de la forme

$$
\partial_{1} \mathbf{A}_{2}-\partial_{2} \mathbf{A}_{1}+\mathbf{A}_{1} \mathbf{A}_{2}-\mathbf{A}_{2} \mathbf{A}_{1}=\mathbf{0} \text { dans } \omega
$$

où $\mathbf{A}_{1}$ et $\mathbf{A}_{2}$ sont des champs de matrices antisymétriques d'ordre trois, qui sont des fonctions des champs $\left(a_{\alpha \beta}\right)$ et $\left(b_{\alpha \beta}\right)$, le champ $\left(a_{\alpha \beta}\right)$ apparaissant en particulier par l'intermédiaire de sa racine carrée. L'immersion inconnue $\boldsymbol{\theta}: \omega \rightarrow \mathbb{R}^{3}$ est trouvée dans cette approche dans des espaces fonctionnels "de faible régularité", à savoir $W_{\mathrm{loc}}^{2, p}\left(\omega ; \mathbb{R}^{3}\right)$, $p>2$. 


\section{INTRODUCTION}

All the notations used, but not defined, in this introduction are defined in the next section.

Greek indices and exponents range in the set $\{1,2\}$. Let $\mathbb{S}^{2}$ denote the space of all symmetric matrices of order two and let $\mathbb{S}_{>}^{2}$ denote the set of all symmetric positive-definite matrices of order two. Let $\omega$ be an open subset of $\mathbb{R}^{2}$ and let $\boldsymbol{\theta} \in \mathscr{C}^{3}\left(\omega ; \mathbb{R}^{3}\right)$ be an immersion. Let

$$
a_{\alpha \beta}=\partial_{\alpha} \boldsymbol{\theta} \cdot \partial_{\beta} \boldsymbol{\theta} \in \mathscr{C}^{2}(\omega) \quad \text { and } \quad b_{\alpha \beta}=\partial_{\alpha \beta} \boldsymbol{\theta} \cdot \frac{\partial_{1} \boldsymbol{\theta} \wedge \partial_{2} \boldsymbol{\theta}}{\left|\partial_{1} \boldsymbol{\theta} \wedge \partial_{2} \theta\right|} \in \mathscr{C}^{1}(\omega)
$$

denote the components of the first and second fundamental forms of the surface $\theta(\omega)$, and let

$$
\Gamma_{\alpha \beta \tau}=\frac{1}{2}\left(\partial_{\beta} a_{\alpha \tau}+\partial_{\alpha} a_{\beta \tau}-\partial_{\tau} a_{\alpha \beta}\right), \quad\left(a^{\sigma \tau}\right)=\left(a_{\alpha \beta}\right)^{-1}, \quad \Gamma_{\alpha \beta}^{\sigma}=a^{\sigma \tau} \Gamma_{\alpha \beta \tau} .
$$

Then it is well known that the functions $a_{\alpha \beta}$ and $b_{\alpha \beta}$ necessarily satisfy compatibility conditions, which take the form of the Gauss and Codazzi-Mainardi equations, viz.,

$$
\begin{aligned}
& \partial_{\beta} \Gamma_{\alpha \sigma \tau}-\partial_{\sigma} \Gamma_{\alpha \beta \tau}+\Gamma_{\alpha \beta}^{\mu} \Gamma_{\sigma \tau \mu}-\Gamma_{\alpha \sigma}^{\mu} \Gamma_{\beta \tau \mu}=b_{\alpha \sigma} b_{\beta \tau}-b_{\alpha \beta} b_{\sigma \tau} \text { in } \omega, \\
& \partial_{\beta} b_{\alpha \sigma}-\partial_{\sigma} b_{\alpha \beta}+\Gamma_{\alpha \sigma}^{\mu} b_{\beta \mu}-\Gamma_{\alpha \beta}^{\mu} b_{\sigma \mu}=0 \text { in } \omega,
\end{aligned}
$$

which in effect simply constitute a re-writing of the relations $\partial_{\alpha \sigma \beta} \boldsymbol{\theta}=\partial_{\alpha \beta \sigma} \boldsymbol{\theta}$. The functions $\Gamma_{\alpha \beta \tau}$ and $\Gamma_{\alpha \beta}^{\sigma}$ are the Christoffel symbols of the first and second kinds associated with the immersion $\boldsymbol{\theta}$.

In fact, the Gauss and Codazzi-Mainardi equations reduce to only three independent equations, since the Gauss equations reduce to only one equation (corresponding, e.g., to $\alpha=1, \beta=2, \sigma=1, \tau=2$ ) and the Codazzi-Mainardi equations reduce to only two equations (corresponding, e.g., to $\alpha=1, \beta=2, \sigma=1$ and $\alpha=1, \beta=2, \sigma=2$ ).

It is also well known that if a field of positive-definite symmetric matrices $\left(a_{\alpha \beta}\right) \in$ $\mathscr{C}^{2}\left(\omega ; \mathbb{S}_{>}^{2}\right)$ and a field of symmetric matrices $\left(b_{\alpha \beta}\right) \in \mathscr{C}^{1}\left(\omega ; \mathbb{S}^{2}\right)$ satisfy the Gauss and Codazzi-Mainardi equations and if the set $\omega$ is simply-connected, then conversely, there exists an immersion $\boldsymbol{\theta} \in \mathscr{C}^{3}\left(\omega ; \mathbb{R}^{3}\right)$ such that $\left(a_{\alpha \beta}\right)$ and $\left(b_{\alpha \beta}\right)$ are the first and second fundamental forms of the surface $\theta(\omega)$.

Furthermore, such an immersion is uniquely defined up to proper isometries of $\mathbb{R}^{3}$. This means that any other immersion $\widetilde{\boldsymbol{\theta}} \in \mathscr{C}^{3}\left(\omega ; \mathbb{R}^{3}\right)$ such that $\left(a_{\alpha \beta}\right)$ and $\left(b_{\alpha \beta}\right)$ are the first and second fundamental forms of the surface $\tilde{\boldsymbol{\theta}}(\omega)$ must be of the form $\widetilde{\boldsymbol{\theta}}(y)=\mathbf{c}+\mathbf{Q} \boldsymbol{\theta}(y)$ for all $y \in \omega$, where $\mathbf{c}$ is a vector in $\mathbb{R}^{3}$ and $\mathbf{Q}$ is a proper orthogonal matrix of order three.

These existence and uniqueness results constitute together the fundamental theorem of surface theory, which goes back to Janet [19] and Cartan [5] (for a self-contained, and essentially elementary, proof, see [12] or [7, Chapter 2]). Its regularity assumptions have since then been significantly weakened: First, Hartman \& Wintner [18] have shown that this theorem still holds if the fields $\left(a_{\alpha \beta}\right)$ and $\left(b_{\alpha \beta}\right)$ are only of class $\mathscr{C}^{1}$ and $\mathscr{C}^{0}$, with a resulting immersion in the space $\mathscr{C}^{2}\left(\omega ; \mathbb{R}^{3}\right)$. Then $S$. Mardare further relaxed these assumptions, first in [20] to fields $\left(a_{\alpha \beta}\right)$ and $\left(b_{\alpha \beta}\right)$ of class $W_{\mathrm{loc}}^{1, \infty}$ and $L_{\mathrm{loc}}^{\infty}$, then in [21] to fields $\left(a_{\alpha \beta}\right)$ and $\left(b_{\alpha \beta}\right)$ of class $W_{\text {loc }}^{1, p}$ and $L_{\text {loc }}^{p}$ for some $p>2$, with resulting immersions in the spaces $W_{\mathrm{loc}}^{2, \infty}\left(\omega ; \mathbb{R}^{3}\right)$ and $W_{\mathrm{loc}}^{2, p}\left(\omega ; \mathbb{R}^{3}\right)$, respectively. Naturally, the Gauss and CodazziMainardi equations are only satisfied in the sense of distributions in such cases.

The main objective of this paper is to identify new compatibility conditions satisfied by the first and second fundamental forms of a surface $\theta(\omega)$ that share the same properties: 
They are necessary, they are sufficient for the existence of the immersion $\boldsymbol{\theta}: \omega \rightarrow \mathbb{R}^{3}$ if $\omega$ is simply-connected, and they hold as well in function spaces with little regularity, corresponding to immersions $\boldsymbol{\theta} \in W_{\mathrm{loc}}^{2, p}\left(\omega ; \mathbb{R}^{3}\right)$ with $p>2$.

Let $\mathbb{M}^{3}$ denote the space of all matrices of order three and let $\mathbb{A}^{3}$ denote the space of all antisymmetric matrices of order three. Then these new compatibility equations, which are first identified in Theorem 3.1 as necessary conditions satisfied by any immersion $\boldsymbol{\theta} \in$ $W_{\text {loc }}^{2, p}\left(\omega ; \mathbb{R}^{3}\right)$, take the following form: The Christoffel symbols $\Gamma_{\alpha \beta \tau} \in L_{\text {loc }}^{p}(\omega)$ and $\Gamma_{\alpha \beta}^{\sigma} \in$ $L_{\text {loc }}^{p}(\omega)$ being defined as above, let $b_{\alpha}^{\sigma}=a^{\beta \sigma} b_{\alpha \beta} \in L_{\text {loc }}^{p}(\omega)$ denote as usual the mixed components of the second fundamental form, and let the matrix fields $\Gamma_{\alpha} \in L_{\mathrm{loc}}^{p}\left(\omega ; \mathbb{M}^{3}\right)$, $\mathbf{C} \in W_{\mathrm{loc}}^{1, p}\left(\omega ; \mathbb{S}_{>}^{3}\right), \mathbf{U} \in W_{\mathrm{loc}}^{1, p}\left(\omega ; \mathbb{S}_{>}^{3}\right)$, and $\mathbf{A}_{\alpha} \in L_{\mathrm{loc}}^{p}\left(\omega ; \mathbb{M}^{3}\right)$ be defined by

$$
\begin{aligned}
\boldsymbol{\Gamma}_{\alpha} & =\left(\begin{array}{llr}
\Gamma_{\alpha 1}^{1} & \Gamma_{\alpha 2}^{1} & -b_{\alpha}^{1} \\
\Gamma_{\alpha 1}^{2} & \Gamma_{\alpha 2}^{2} & -b_{\alpha}^{2} \\
b_{\alpha 1} & b_{\alpha 2} & 0
\end{array}\right), \quad \mathbf{C}=\left(\begin{array}{rrr}
a_{11} & a_{12} & 0 \\
a_{21} & a_{22} & 0 \\
0 & 0 & 1
\end{array}\right), \\
\mathbf{U} & =\mathbf{C}^{1 / 2}, \quad \mathbf{A}_{\alpha}=\left(\mathbf{U} \boldsymbol{\Gamma}_{\alpha}-\partial_{\alpha} \mathbf{U}\right) \mathbf{U}^{-1} .
\end{aligned}
$$

Then the matrix fields $\mathbf{A}_{\alpha}$ are antisymmetric and their components necessarily satisfy three compatibility conditions that take the form of the following matrix equation:

$$
\partial_{1} \mathbf{A}_{2}-\partial_{2} \mathbf{A}_{1}+\mathbf{A}_{1} \mathbf{A}_{2}-\mathbf{A}_{2} \mathbf{A}_{1}=\mathbf{0} \text { in } \mathscr{D}^{\prime}\left(\omega ; \mathbb{A}^{3}\right) .
$$

We then establish in Theorem 5.1 the main result of this paper, namely that these compatibility conditions are also sufficient: Under the assumption that the open set $\omega \subset \mathbb{R}^{2}$ is simply-connected, we show that, if for some $p>2$ a field of positive-definite symmetric matrices $\left(a_{\alpha \beta}\right) \in W_{\mathrm{loc}}^{1, p}\left(\omega ; \mathbb{S}_{>}^{2}\right)$ and a field of symmetric matrices $\left(b_{\alpha \beta}\right) \in L_{\mathrm{loc}}^{p}\left(\omega ; \mathbb{S}^{2}\right)$ satisfy the matrix equation

$$
\partial_{1} \mathbf{A}_{2}-\partial_{2} \mathbf{A}_{1}+\mathbf{A}_{1} \mathbf{A}_{2}-\mathbf{A}_{2} \mathbf{A}_{1}=\mathbf{0} \text { in } \mathscr{D}^{\prime}\left(\omega ; \mathbb{A}^{3}\right),
$$

where the matrix fields $\mathbf{A}_{\alpha} \in L_{\text {loc }}^{p}\left(\omega ; \mathbb{A}^{3}\right)$ are constructed as above from the matrix fields $\left(a_{\alpha \beta}\right)$ and $\left(b_{\alpha \beta}\right)$, then there exists an immersion $\boldsymbol{\theta} \in W_{\mathrm{loc}}^{2, p}\left(\omega ; \mathbb{R}^{3}\right)$, unique up to proper isometries of $\mathbb{R}^{3}$, such that

$$
a_{\alpha \beta}=\partial_{\alpha} \boldsymbol{\theta} \cdot \partial_{\beta} \boldsymbol{\theta} \text { in } W_{\mathrm{loc}}^{1, p}(\omega) \quad \text { and } \quad b_{\alpha \beta}=\partial_{\alpha \beta} \boldsymbol{\theta} \cdot \frac{\partial_{1} \boldsymbol{\theta} \wedge \partial_{2} \boldsymbol{\theta}}{\left|\partial_{1} \boldsymbol{\theta} \wedge \partial_{2} \boldsymbol{\theta}\right|} \text { in } L_{\mathrm{loc}}^{p}(\omega) .
$$

The proof consists first in determining a proper orthogonal matrix field $\mathbf{R}$ of class $W_{\mathrm{loc}}^{1, p}$ in $\omega$ by solving the Pfaff system $\partial_{\alpha} \mathbf{R}=\mathbf{R} \mathbf{A}_{\alpha}$, second in determining an immersion $\boldsymbol{\theta} \in$ $W_{\text {loc }}^{2, p}\left(\omega ; \mathbb{R}^{3}\right)$ by solving the equations $\partial_{\alpha} \boldsymbol{\theta}=\mathbf{R} \mathbf{u}_{\alpha}$, where $\mathbf{u}_{\alpha}$ denotes the $\alpha$-th column vector field of the matrix field $\mathbf{U}=\mathbf{C}^{1 / 2}$, and third, in showing that $\left(a_{\alpha \beta}\right)$ and $\left(b_{\alpha \beta}\right)$ are indeed the first and second fundamental forms of the surface $\theta(\omega)$.

By contrast, the proof in the "classical" approach (once properly extended to spaces with little regularity; cf. S. Mardare [21]) first seeks a matrix field $\mathbf{F} \in W_{\mathrm{loc}}^{1, p}\left(\omega ; \mathbb{M}^{3}\right)$ as a solution of the Pfaff system $\partial_{\alpha} \mathbf{F}=\mathbf{F} \boldsymbol{\Gamma}_{\alpha}$, then the sought immersion $\boldsymbol{\theta} \in W_{\mathrm{loc}}^{2, p}\left(\omega ; \mathbb{R}^{3}\right)$ as a solution to the system $\partial_{\alpha} \boldsymbol{\theta}=\mathbf{f}_{\alpha}$, where $\mathbf{f}_{\alpha}$ denotes the $\alpha$-th column vector field of the matrix field $\mathbf{F}$.

We emphasize that our existence result is global and that it holds in function spaces with little regularity, viz., $W_{\text {loc }}^{2, p}\left(\omega ; \mathbb{R}^{3}\right)$, thanks to deep existence results for Pfaff systems and Poincaré's lemma (recalled in Theorems 4.1 and 4.2) recently obtained by S. Mardare, 
first in $\mathbb{R}^{2}$ (cf. [21]), then in $\mathbb{R}^{N}$ for an arbitrary dimension $N \geq 2$ (cf. [22]). Note also that, as observed in [22], such regularities are optimal.

An inspection of the proof reveals the geometric nature of this approach: Let the canonical three-dimensional extension $\Theta: \omega \times \mathbb{R} \rightarrow \mathbb{R}^{3}$ of an immersion $\boldsymbol{\theta} \in W_{\mathrm{loc}}^{2, p}\left(\omega ; \mathbb{R}^{3}\right)$ be defined by

$$
\boldsymbol{\Theta}\left(y, x_{3}\right)=\boldsymbol{\theta}(y)+x_{3} \mathbf{a}_{3}(y), \text { for all } y \in \omega \text { and } x_{3} \in \mathbb{R},
$$

where $\mathbf{a}_{3}=\frac{\mathbf{a}_{1} \times \mathbf{a}_{2}}{\left|\mathbf{a}_{1} \times \mathbf{a}_{2}\right|}$ and $\mathbf{a}_{\alpha}=\partial_{\alpha} \boldsymbol{\theta}$, and let the matrix field $\mathbf{F} \in W_{\mathrm{loc}}^{1, p}\left(\omega ; \mathbb{M}^{3}\right)$ be defined by $\mathbf{F}(y)=\nabla \boldsymbol{\Theta}(y, 0)$. Then the fields $\mathbf{R}$ and $\mathbf{U}$ satisfy

$$
\mathbf{F}=\mathbf{R} \mathbf{U} \text { in } W_{\mathrm{loc}}^{1, p}\left(\omega ; \mathbb{M}^{3}\right) \text {. }
$$

In other words, the proper orthogonal matrix field $\mathbf{R}$ is nothing but the rotation field that appears in the polar factorization of the gradient of the canonical three-dimensional extension $\Theta$ of the immersion $\boldsymbol{\theta}$ at $x_{3}=0$.

The above compatibility conditions are in a sense the "surface analogs" of similar "three-dimensional" compatibility conditions satisfied in an open subset $\Omega$ of $\mathbb{R}^{3}$ by the square root of the metric tensor field $\nabla \Theta^{T} \nabla \Theta \in \mathscr{C}^{2}\left(\Omega ; \mathbb{S}_{>}^{3}\right)$ associated with a given immersion $\Theta \in \mathscr{C}^{3}\left(\Omega ; \mathbb{R}^{3}\right)$. These three-dimensional conditions, which were first identified (in componentwise form) by Shield [26], have been recently shown to be also sufficient for the existence of such an immersion $\Theta$ when the set $\Omega$ is simply-connected, also in function spaces with little regularity; cf. [11].

We conclude this paper by showing in Theorem 6.1 that these new compatibility conditions are, as expected, equivalent to the Gauss and Codazzi-Mainardi equations.

As advocated notably by Simmonds \& Danielson [27], Valid [31], Pietraszkiewicz [23, 24], Pietraszkiewicz \& Badur [25], Başar [4], Simo \& Fox [28], or Galka \& Telega [16] among others, rotation fields can be advantageously introduced as bona fide unknowns in the mathematical modeling and numerical simulation of nonlinearly elastic shells. The present study may thus be viewed as a first step towards the mathematical justification of such an approach.

This viewpoint is thus analogous to that of Antman [3], who, back in 1976, was the first to suggest that the metric tensor field of a deformed configuration in nonlinear threedimensional elasticity could be considered as the primary unknown on its own, instead of the position vector field as is customary. It was likewise, but more recently, recognized that the first and second fundamental form of a deformed middle surface in nonlinear shell theory could be considered as primary unknowns on their own, instead of the position vector field of the middle surface (for recent developments and references on such approaches see [14] and [9]).

The results of this paper have been announced in [10].

\section{NOTATIONS AND PRELIMINARIES}

This section gathers various conventions, notations, definitions, and preliminary results that will be used throughout the article.

Greek indices and exponents range in the set $\{1,2\}$ and the summation convention with respect to repeated indices or exponents is used in conjunction with this rule.

All matrices considered in this paper are real. The notations $\mathbb{M}^{n}, \mathbb{M}_{+}^{n}, \mathbb{S}^{n}, \mathbb{S}_{>}^{n}, \mathbb{A}^{n}$, and $\mathbb{O}_{+}^{n}$ respectively designate the sets of all square matrices of order $n$, of all matrices $\mathbf{F} \in \mathbb{M}^{n}$ with $\operatorname{det} \mathbf{F}>0$, of all symmetric matrices, of all positive-definite symmetric matrices, of all antisymmetric matrices, and of all proper orthogonal matrices, i.e., orthogonal matrices 
$\mathbf{Q}$ with $\operatorname{det} \mathbf{Q}=1$, of order $n$. The notation $\mathbb{M}^{m \times n}$ designates the space of all matrices with $m$ rows and $n$ columns. When it is identified with a matrix, a vector in $\mathbb{R}^{n}$ is always understood as a column vector, i.e., a matrix in $\mathbb{M}^{n \times 1}$. Given a matrix $\mathbf{A} \in \mathbb{M}^{n},[\mathbf{A}]_{j}$ denotes its $j$-th column vector.

The Euclidean norm of $\mathbf{a} \in \mathbb{R}^{n}$, the Euclidean inner-product of $\mathbf{a}, \mathbf{b} \in \mathbb{R}^{n}$, and the vector product of $\mathbf{a}, \mathbf{b} \in \mathbb{R}^{n}$ are respectively denoted $|\mathbf{a}|, \mathbf{a} \cdot \mathbf{b}$, and $\mathbf{a} \wedge \mathbf{b}$.

Given any matrix $\mathbf{C} \in \mathbb{S}_{>}^{n}$, there exists one and only one matrix $\mathbf{U} \in \mathbb{S}_{>}^{n}$ such that $\mathbf{U}^{2}=\mathbf{C}$ (for a proof, see, e.g., [6, Theorem 3.2-1]). The matrix $\mathbf{U}$ is denoted $\mathbf{C}^{1 / 2}$ and is called the square root of $\mathbf{C}$. The mapping $\mathbf{C} \in \mathbb{S}_{>}^{n} \mapsto \mathbf{C}^{1 / 2} \in \mathbb{S}_{>}^{n}$ defined in this fashion is of class $\mathbf{C}^{\infty}$ (for a proof, see, e.g., Gurtin [17, Section 13]). Clearly, the mapping $\mathbf{A} \in \mathbb{S}_{>}^{n} \mapsto \mathbf{A}^{-1} \in \mathbb{S}_{>}^{n}$ is also of class $\mathscr{C}^{\infty}$.

Any invertible matrix $\mathbf{F} \in \mathbb{M}_{+}^{n}$ admits a unique polar factorization $\mathbf{F}=\mathbf{R} \mathbf{U}$, as a product of a matrix $\mathbf{R} \in \mathbb{O}_{+}^{n}$ by a matrix $\mathbf{U} \in \mathbb{S}_{>}^{n}$, with $\mathbf{U}=\left(\mathbf{F}^{T} \mathbf{F}\right)^{1 / 2}$ and $\mathbf{R}=\mathbf{F} \mathbf{U}^{-1}$ (for a proof, see, e.g., [6, Theorem 3.2-2]).

The coordinates of a point $y \in \mathbb{R}^{2}$ are denoted $y_{\alpha}$ and partial derivatives of the first and second order, in the usual sense or in the sense of distributions, are denoted $\partial_{\alpha}:=\partial / \partial y_{\alpha}$ and $\partial_{\alpha \beta}:=\partial^{2} / \partial y_{\alpha} \partial y_{\beta}$.

An open subset $\Omega$ of $\mathbb{R}^{n}$ is simply-connected if, as a topological space, it is arcwise connected and any closed loop in $\Omega$ is homotopic to a point.

All the function spaces considered in this paper are over $\mathbb{R}$. Let $\omega$ be an open subset of $\mathbb{R}^{2}$. The notation $\chi \Subset \omega$ means that $\bar{\chi}$ is a compact subset of $\omega$. The notations $\mathscr{D}(\omega)$ and $\mathscr{D}^{\prime}(\omega)$ respectively designate the space of all functions infinitely differentiable on $\omega$ whose support is a compact subset of $\omega$ and the space of distributions over $\omega$. The notations $\mathscr{C}^{m}(\omega), W^{m, p}(\omega)$, and $W_{\text {loc }}^{m, p}(\omega)$ designate the usual spaces of continuous (for $m=0$ ) and continuously differentiable (for $m \geq 1$ ) functions and the usual Sobolev spaces, with $W^{0, p}(\omega)=L^{p}(\omega)$ and $W_{\mathrm{loc}}^{0, p}(\omega)=L_{\mathrm{loc}}^{p}(\omega)$.

Let $\mathbb{X}$ denote a finite-dimensional space, such as $\mathbb{R}^{n}, \mathbb{M}^{n}$, etc., or a subset thereof, such as $\mathbb{S}_{>}^{n}, \mathbb{O}_{+}^{n}$, etc. Then notations such as $W_{\text {loc }}^{1, p}(\omega ; \mathbb{X}), \mathscr{D}^{\prime}(\omega ; \mathbb{X})$, etc., designate spaces or sets of vector fields or matrix fields with values in $\mathbb{X}$ and whose components belong to $W_{\text {loc }}^{1, p}(\omega), \mathscr{D}^{\prime}(\omega)$, etc.

Let $\omega$ be an open subset of $\mathbb{R}^{2}$. Although an element $f$ in the space $W_{\text {loc }}^{1, p}(\omega)$, where $p>$ 2 , is an equivalence class of functions, it will be systematically identified with a function $f \in \mathscr{C}^{0}(\omega)$, in view of the Sobolev imbeddings $W^{1, p}(\beta) \subset \mathscr{C}^{0}(\bar{\beta})$ that hold for all open balls $\beta \Subset \omega$. Likewise, an element $f$ in $W_{\text {loc }}^{2, p}(\omega)$ will be identified with a function $f \in$ $\mathscr{C}^{1}(\omega)$. We also note that, for any $p>2, f g \in L_{\mathrm{loc}}^{p}(\omega)$ if $f \in W_{\mathrm{loc}}^{1, p}(\omega)$ and $g \in L_{\mathrm{loc}}^{p}(\omega)$, and that $f g \in W_{\text {loc }}^{1, p}(\omega)$ if $f \in W_{\text {loc }}^{1, p}(\omega)$ and $g \in W_{\text {loc }}^{1, p}(\omega)$, since $W^{1, p}(\beta)$ is an algebra for all open balls $\beta \Subset \omega$.

Finally, we recall that a mapping $\theta \in \mathscr{C}^{1}\left(\omega ; \mathbb{R}^{3}\right)$, where $\omega$ is again an open subset of $\mathbb{R}^{2}$, is an immersion if the two vectors $\partial_{\alpha} \theta(y)$ are linearly independent for all $y \in \omega$.

\section{NEW COMPATIBILITY CONDITIONS SATISFIED BY THE FIRST AND SECOND FUNDAMENTAL FORMS OF A GIVEN SURFACE}

Our first task naturally consists in identifying the announced compatibility conditions as necessary conditions.

Theorem 3.1. Let $\omega$ be an open subset of $\mathbb{R}^{2}$, let $p>2$, and let $\boldsymbol{\theta} \in W_{\mathrm{loc}}^{2, p}\left(\omega ; \mathbb{R}^{3}\right)$ be an immersion. Define the vector fields $\mathbf{a}_{i} \in W_{\mathrm{loc}}^{1, p}\left(\omega ; \mathbb{R}^{3}\right), 1 \leq i \leq 3$, and the matrix fields 


$$
\begin{aligned}
& \left(a_{\alpha \beta}\right) \in W_{\mathrm{loc}}^{1, p}\left(\omega ; \mathbb{S}_{>}^{2}\right) \text { and }\left(b_{\alpha \beta}\right) \in L_{\mathrm{loc}}^{p}\left(\omega ; \mathbb{S}^{2}\right) \text { by } \\
& \mathbf{a}_{\alpha}:=\partial_{\alpha} \boldsymbol{\theta} \quad \text { and } \quad \mathbf{a}_{3}:=\frac{\mathbf{a}_{1} \wedge \mathbf{a}_{2}}{\left|\mathbf{a}_{1} \wedge \mathbf{a}_{2}\right|}, \\
& a_{\alpha \beta}:=\mathbf{a}_{\alpha} \cdot \mathbf{a}_{\beta} \quad \text { and } \quad b_{\alpha \beta}:=\partial_{\alpha} \mathbf{a}_{\beta} \cdot \mathbf{a}_{3} .
\end{aligned}
$$

Define also the matrix fields $\left(a^{\sigma \tau}\right) \in W_{\mathrm{loc}}^{1, p}\left(\omega ; \mathbb{S}_{>}^{2}\right)$ and the functions $\Gamma_{\alpha \beta \tau} \in L_{\mathrm{loc}}^{p}(\omega), \Gamma_{\alpha \beta}^{\sigma} \in$ $L_{\mathrm{loc}}^{p}(\omega)$, and $b_{\alpha}^{\sigma} \in L_{\mathrm{loc}}^{p}(\omega)$ by

$$
\begin{aligned}
& \left(a^{\sigma \tau}\right):=\left(a_{\alpha \beta}\right)^{-1}, \quad \Gamma_{\alpha \beta \tau}:=\frac{1}{2}\left(\partial_{\beta} a_{\alpha \tau}+\partial_{\alpha} a_{\beta \tau}-\partial_{\tau} a_{\alpha \beta}\right), \\
& \Gamma_{\alpha \beta}^{\sigma}:=a^{\sigma \tau} \Gamma_{\alpha \beta \tau}, \quad b_{\alpha}^{\sigma}:=a^{\beta \sigma} b_{\alpha \beta} .
\end{aligned}
$$

Finally, define the matrix fields $\Gamma_{\alpha} \in L_{\mathrm{loc}}^{p}\left(\omega ; \mathbb{M}^{3}\right), \mathbf{C} \in W_{\mathrm{loc}}^{1, p}\left(\omega ; \mathbb{S}_{>}^{3}\right), \mathbf{U} \in W_{\mathrm{loc}}^{1, p}\left(\omega ; \mathbb{S}_{>}^{3}\right)$, and $\mathbf{A}_{\alpha} \in L_{\mathrm{loc}}^{p}\left(\omega ; \mathbb{M}^{3}\right)$ by

$$
\begin{aligned}
& \Gamma_{\alpha}:=\left(\begin{array}{llr}
\Gamma_{\alpha 1}^{1} & \Gamma_{\alpha 2}^{1} & -b_{\alpha}^{1} \\
\Gamma_{\alpha 1}^{2} & \Gamma_{\alpha 2}^{2} & -b_{\alpha}^{2} \\
b_{\alpha 1} & b_{\alpha 2} & 0
\end{array}\right), \quad \mathbf{C}:=\left(\begin{array}{rrr}
a_{11} & a_{12} & 0 \\
a_{21} & a_{22} & 0 \\
0 & 0 & 1
\end{array}\right), \\
& \mathbf{U}:=\mathbf{C}^{1 / 2}, \quad \mathbf{A}_{\alpha}:=\left(\mathbf{U} \Gamma_{\alpha}-\partial_{\alpha} \mathbf{U}\right) \mathbf{U}^{-1} .
\end{aligned}
$$

Then the matrix fields $\mathbf{A}_{\alpha}$ are antisymmetric and they necessarily satisfy compatibility conditions that take the form of the following matrix equation:

$$
\partial_{1} \mathbf{A}_{2}-\partial_{2} \mathbf{A}_{1}+\mathbf{A}_{1} \mathbf{A}_{2}-\mathbf{A}_{2} \mathbf{A}_{1}=\mathbf{0} \text { in } \mathscr{D}^{\prime}\left(\omega ; \mathbb{A}^{3}\right) .
$$

Proof. (i) Technical preliminaries. Recall that any function in $W_{\text {loc }}^{1, p}(\omega)$ is identified with a function in $\mathscr{C}^{0}(\omega)$ (cf. Section 2). Given any open ball $\beta \Subset \omega$, there thus exists a constant $c(\beta)>0$ such that

$$
\left|\mathbf{a}_{1}(y) \wedge \mathbf{a}_{2}(y)\right| \geq c(\beta) \text { and } \operatorname{det}\left(a_{\alpha \beta}(y)\right) \geq c(\beta) \text { for all } y \in \bar{\beta} .
$$

Consequently, the vector $\mathbf{a}_{3}(y)$ and the matrix $\left(a^{\sigma \tau}(y)\right)$ are well defined for all $y \in \bar{\beta}$. That the components of the vector field $\mathbf{a}_{3}: \bar{\beta} \rightarrow \mathbb{R}^{3}$ and of the matrix field $\left(a^{\sigma \tau}\right): \bar{\beta} \rightarrow \mathbb{S}_{>}^{2}$ defined in this fashion belong to the space $W_{\text {loc }}^{1, p}(\omega)$ then simply follows from their explicit expressions in terms of the components of the vector fields $\mathbf{a}_{\alpha}$ and of the matrix field $\left(a_{\alpha \beta}\right)$ and from the property that $W^{1, p}(\beta)$ is an algebra for $p>2$. We thus have

$$
\mathbf{a}_{3} \in W_{\mathrm{loc}}^{1, p}\left(\omega ; \mathbb{R}^{3}\right) \quad \text { and } \quad\left(a^{\sigma \tau}\right) \in W_{\mathrm{loc}}^{1, p}\left(\omega ; \mathbb{S}_{>}^{2}\right) .
$$

Since the mappings $\mathbf{C} \in \mathbb{S}_{>}^{3} \rightarrow \mathbf{C}^{1 / 2} \in \mathbb{S}_{>}^{3}$ and $\mathbf{U} \in \mathbb{S}_{>}^{3} \rightarrow \mathbf{U}^{-1} \in \mathbb{S}_{>}^{3}$ are both of class $\mathscr{C}^{\infty}$, analogous arguments likewise show that the matrix fields $\mathbf{U}$ and $\mathbf{U}^{-1}$ are well defined and they satisfy

$$
\mathbf{U} \in W_{\mathrm{loc}}^{1, p}\left(\omega ; \mathbb{S}_{>}^{3}\right) \quad \text { and } \quad \mathbf{U}^{-1} \in W_{\mathrm{loc}}^{1, p}\left(\omega ; \mathbb{S}_{>}^{3}\right) .
$$

The definitions of the functions $a_{\alpha \beta}, \Gamma_{\alpha \beta \tau}$, and $\Gamma_{\alpha \beta}^{\sigma}$ immediately imply that $\Gamma_{\alpha \beta \tau}=$ $\partial_{\alpha} \mathbf{a}_{\beta} \cdot \mathbf{a}_{\tau}$ and $\Gamma_{\alpha \beta}^{\sigma}=\partial_{\alpha} \mathbf{a}_{\beta} \cdot \mathbf{a}^{\sigma}$, where $\mathbf{a}^{\sigma}:=a^{\sigma \tau} \mathbf{a}_{\tau}$. Together with the definitions of the functions $b_{\alpha \beta}$ and $b_{\alpha}^{\sigma}$, these relations in turn imply that

$$
\partial_{\alpha} \mathbf{a}_{\beta}=\Gamma_{\alpha \beta}^{\sigma} \mathbf{a}_{\sigma}+b_{\alpha \beta} \mathbf{a}_{3} \quad \text { and } \quad \partial_{\alpha} \mathbf{a}_{3}=-b_{\alpha}^{\sigma} \mathbf{a}_{\sigma} \text { in } L_{\mathrm{loc}}^{p}\left(\omega ; \mathbb{R}^{3}\right) .
$$

Of course, these relations are nothing but the extensions of the classical formulas of Gauss and Weingarten to function spaces with little regularity. 
(ii) Introduction of the antisymmetric matrix fields $\mathbf{A}_{\alpha}$. To begin with, we note that the matrix field $\mathbf{F} \in W_{\mathrm{loc}}^{1, p}\left(\omega ; \mathbb{M}_{+}^{3}\right)$ defined by $[\mathbf{F}]_{j}:=\mathbf{a}_{j}, 1 \leq j \leq 3$, satisfies

$$
\mathbf{C}=\mathbf{F}^{T} \mathbf{F} \text { in } W_{\mathrm{loc}}^{1, p}\left(\omega ; \mathbb{S}_{>}^{3}\right) \quad \text { and } \quad \partial_{\alpha} \mathbf{F}=\mathbf{F} \boldsymbol{\Gamma}_{\alpha} \quad \text { in } L_{\mathrm{loc}}^{p}\left(\omega ; \mathbb{M}^{3}\right)
$$

The first relation immediately follows from the relations $\mathbf{a}_{\alpha} \cdot \mathbf{a}_{\beta}=a_{\alpha \beta}$ and $\mathbf{a}_{i} \cdot \mathbf{a}_{3}=\delta_{i 3}$. The definition of the vector field $\mathbf{a}_{3}$ in terms of the vector fields $\mathbf{a}_{\alpha}$ also shows that $\mathbf{F}(y) \in \mathbb{M}_{+}^{3}$ for all $y \in \omega$. The second relation is simply a convenient rewriting in matrix form of the formulas of Gauss and Weingarten, based on the relations

$$
\left[\mathbf{F} \Gamma_{\alpha}\right]_{\beta}=\partial_{\alpha} \mathbf{a}_{\beta} \quad \text { and } \quad\left[\mathbf{F} \boldsymbol{\Gamma}_{\alpha}\right]_{3}=\partial_{\alpha} \mathbf{a}_{3},
$$

which themselves follow from the definition of the matrix fields $\mathbf{F}$ and $\Gamma_{\alpha}$ (this observation is due to S. Mardare [20]).

At each point $y \in \omega$, let $\mathbf{F}(y)=\mathbf{R}(y) \mathbf{U}(y)$ denote the polar factorization (Section 2) of the matrix $\mathbf{F}(y) \in \mathbb{M}_{+}^{3}$, with

$$
\mathbf{U}(y):=\left(\mathbf{F}^{T}(y) \mathbf{F}(y)\right)^{1 / 2} \in \mathbb{S}_{>}^{3} \quad \text { and } \quad \mathbf{R}(y)=\mathbf{F}(y) \mathbf{U}(y)^{-1} \in \mathbb{O}_{+}^{3} .
$$

Since $\mathbf{U} \in W_{\text {loc }}^{1, p}\left(\omega ; \mathbb{S}_{>}^{3}\right)$ as already noted in part (i), it follows that

$$
\mathbf{R} \in W_{\mathrm{loc}}^{1, p}\left(\omega ; \mathbb{O}_{+}^{3}\right) .
$$

Noting that the polar factorization $\mathbf{F}=\mathbf{R} \mathbf{U}$ implies that

$$
\partial_{\alpha} \mathbf{F}=\mathbf{R} \mathbf{U} \Gamma_{\alpha}=\left(\partial_{\alpha} \mathbf{R}\right) \mathbf{U}+\mathbf{R} \partial_{\alpha} \mathbf{U}
$$

and that the matrices $\mathbf{U}(y)$ are invertible at all $y \in \omega$, we obtain

$$
\partial_{\alpha} \mathbf{R}=\mathbf{R} \mathbf{A}_{\alpha} \text { in } L_{\mathrm{loc}}^{p}\left(\omega ; \mathbb{M}^{3}\right) \text { where } \mathbf{A}_{\alpha}:=\left(\mathbf{U} \Gamma_{\alpha}-\partial_{\alpha} \mathbf{U}\right) \mathbf{U}^{-1} \in L_{\mathrm{loc}}^{p}\left(\omega ; \mathbb{M}^{3}\right)
$$

The relations $\mathbf{I}=\mathbf{R}^{T} \mathbf{R}$ and $\partial_{\alpha} \mathbf{R}=\mathbf{R} \mathbf{A}_{\alpha}$ then imply that

$$
\mathbf{0}=\left(\partial_{\alpha} \mathbf{R}\right)^{T} \mathbf{R}+\mathbf{R}^{T} \partial_{\alpha} \mathbf{R}=\mathbf{A}_{\alpha}^{T}+\mathbf{A}_{\alpha} \text { in } \omega,
$$

which shows that the matrix fields $\mathbf{A}_{\alpha}$ are antisymmetric.

(iii) Compatibility relations satisfied by the matrix fields $\mathbf{A}_{\alpha}$. In what follows, $p^{\prime}:=$ $\frac{p}{p-1}$ designates the conjugate exponent of $p$ and $X^{\prime}\langle\cdot, \cdot\rangle_{X}$ designates the duality pairing between a topologigal vector space $X$ and its dual $X^{\prime}$. For notational brevity, spaces such as $\mathscr{D}\left(\omega ; \mathbb{M}^{3}\right), W_{0}^{1, p}\left(\omega ; \mathbb{M}^{3}\right)$, etc., appearing in duality pairings will be abbreviated as $\mathscr{D}(\omega)$, $W_{0}^{1, p}(\omega)$, etc.

Given matrix fields $\mathbf{R} \in W_{\mathrm{loc}}^{1, p}\left(\omega ; \mathbb{M}^{3}\right)$ and $\mathbf{A} \in L_{\mathrm{loc}}^{p}\left(\omega ; \mathbb{M}^{3}\right)$, the distribution $\mathbf{R A} \in \mathscr{D}^{\prime}\left(\omega ; \mathbb{M}^{3}\right)$ is well defined since $\mathbf{R A} \in L_{\text {loc }}^{p}\left(\omega ; \mathbb{M}^{3}\right)$. Likewise, each distribution $\mathbf{R} \partial_{\alpha} \mathbf{A} \in \mathscr{D}^{\prime}\left(\omega ; \mathbb{M}^{3}\right)$ is well defined by the relations

$$
\mathscr{D}^{\prime}(\omega)\left\langle\mathbf{R} \partial_{\alpha} \mathbf{A}, \boldsymbol{\varphi}\right\rangle_{\mathscr{D}(\omega)}:={ }_{W^{-1, p^{\prime}}(\chi)}\left\langle\partial_{\alpha} \mathbf{A}, \mathbf{R}^{T} \boldsymbol{\varphi}\right\rangle_{W_{0}^{1, p}(\chi)} \text { for all } \boldsymbol{\varphi} \in \mathscr{D}\left(\omega ; \mathbb{M}^{3}\right),
$$

where $\chi$ designates the interior of the support of $\varphi$.

The relations $\partial_{\alpha} \mathbf{R}=\mathbf{R} \mathbf{A}_{\alpha}$ in $L_{\mathrm{loc}}^{p}\left(\omega ; \mathbb{M}^{3}\right)$ satisfied by the matrix fields $\mathbf{R} \in W_{\mathrm{loc}}^{1, p}\left(\omega ; \mathbb{O}_{+}^{3}\right)$ and $\mathbf{A}_{\alpha} \in L_{\text {loc }}^{p}\left(\omega ; \mathbb{A}^{3}\right)$ found in part (ii) imply that

$$
\begin{aligned}
& \partial_{\beta \alpha} \mathbf{R}=\left(\partial_{\beta} \mathbf{R}\right) \mathbf{A}_{\alpha}+\mathbf{R} \partial_{\beta} \mathbf{A}_{\alpha}=\mathbf{R} \mathbf{A}_{\beta} \mathbf{A}_{\alpha}+\mathbf{R} \partial_{\beta} \mathbf{A}_{\alpha} \text { in } \mathscr{D}^{\prime}\left(\omega ; \mathbb{M}^{3}\right), \\
& \partial_{\alpha \beta} \mathbf{R}=\left(\partial_{\alpha} \mathbf{R}\right) \mathbf{A}_{\beta}+\mathbf{R} \partial_{\alpha} \mathbf{A}_{\beta}=\mathbf{R} \mathbf{A}_{\alpha} \mathbf{A}_{\beta}+\mathbf{R} \partial_{\alpha} \mathbf{A}_{\beta} \text { in } \mathscr{D}^{\prime}\left(\omega ; \mathbb{M}^{3}\right)
\end{aligned}
$$


(the products $\mathbf{R} \mathbf{A}_{\beta} \mathbf{A}_{\alpha}$ are well-defined distributions, since $\mathbf{A}_{\beta} \mathbf{A}_{\alpha} \in L_{\text {loc }}^{p / 2}\left(\omega ; \mathbb{M}^{3}\right)$ ). Hence the relations $\partial_{\beta \alpha} \mathbf{R}=\partial_{\alpha \beta} \mathbf{R}$ imply that

$$
\mathbf{R} \partial_{\alpha} \mathbf{A}_{\beta}-\mathbf{R} \partial_{\beta} \mathbf{A}_{\alpha}+\mathbf{R} \mathbf{A}_{\alpha} \mathbf{A}_{\beta}-\mathbf{R} \mathbf{A}_{\beta} \mathbf{A}_{\alpha}=\mathbf{0} \text { in } \mathscr{D}^{\prime}\left(\omega ; \mathbb{M}^{3}\right) .
$$

Consequently,

$$
{ }_{W^{-1, p^{\prime}}(\chi)}\left\langle\partial_{\alpha} \mathbf{A}_{\beta}-\partial_{\beta} \mathbf{A}_{\alpha}+\mathbf{A}_{\alpha} \mathbf{A}_{\beta}-\mathbf{A}_{\beta} \mathbf{A}_{\alpha}, \mathbf{R}^{T} \boldsymbol{\varphi}\right\rangle_{W_{0}^{1, p}(\chi)}=0 \text { for all } \boldsymbol{\varphi} \in D\left(\omega ; \mathbb{M}^{3}\right) .
$$

The matrices $\mathbf{R}^{T}(y)$ being invertible at each $y \in \omega$, any matrix field $\psi \in D\left(\omega ; \mathbb{M}^{3}\right)$ can be written as $\psi=\mathbf{R}^{T} \boldsymbol{\varphi}$ with $\varphi \in \mathscr{D}\left(\omega ; \mathbb{M}^{3}\right)$ and the fields $\psi$ and $\varphi$ have the same support. Consequently,

$$
\begin{aligned}
& W^{-1, p^{\prime}(\chi)}\left\langle\partial_{\alpha} \mathbf{A}_{\beta}-\partial_{\beta} \mathbf{A}_{\alpha}+\mathbf{A}_{\alpha} \mathbf{A}_{\beta}-\mathbf{A}_{\beta} \mathbf{A}_{\alpha}, \mathbf{R}^{T} \boldsymbol{\varphi}\right\rangle_{W_{0}^{1, p}(\chi)} \\
& \quad=\mathscr{D}^{\prime}(\omega) \\
& \left\langle\partial_{\alpha} \mathbf{A}_{\beta}-\partial_{\beta} \mathbf{A}_{\alpha}+\mathbf{A}_{\alpha} \mathbf{A}_{\beta}-\mathbf{A}_{\beta} \mathbf{A}_{\alpha}, \psi\right\rangle_{\mathscr{D}(\omega)}=0
\end{aligned}
$$

for all $\psi \in \mathscr{D}\left(\omega ; \mathbb{M}^{3}\right)$ and thus

$$
\partial_{\alpha} \mathbf{A}_{\beta}-\partial_{\beta} \mathbf{A}_{\alpha}+\mathbf{A}_{\alpha} \mathbf{A}_{\beta}-\mathbf{A}_{\beta} \mathbf{A}_{\alpha}=\mathbf{0} \text { in } \mathscr{D}^{\prime}\left(\omega ; \mathbb{A}^{3}\right) .
$$

In order that these relations hold for all $\alpha, \beta \in\{1,2\}$, it clearly suffices that the relation corresponding to $\alpha=1$ and $\beta=2$ holds.

Several comments about this result are in order: First, the various functions $a_{\alpha \beta}, a^{\sigma \tau}$, $b_{\alpha \beta}, b_{\alpha}^{\sigma}, \Gamma_{\alpha \beta \tau}$, and $\Gamma_{\alpha \beta}^{\sigma}$ are all familiar. They respectively represent the covariant and contravariant components of the first fundamental form and the covariant and mixed components of the second fundamental form of the surface $\theta(\omega)$, and the associated Christoffel symbols of the first and second kinds. Their specific expressions, together with those of the matrix fields $\boldsymbol{\Gamma}_{\alpha}, \mathbf{C}, \mathbf{U}$, and $\mathbf{A}_{\alpha}$, show that the matrix equation $\partial_{1} \mathbf{A}_{2}-\partial_{2} \mathbf{A}_{1}+\mathbf{A}_{1} \mathbf{A}_{2}-$ $\mathbf{A}_{2} \mathbf{A}_{1}=\mathbf{0}$ in $\mathscr{D}^{\prime}\left(\omega ; \mathbb{A}^{3}\right)$ is indeed a set of compatibility conditions involving only the components of the first and second fundamental forms of the surface $\boldsymbol{\theta}(\omega)$.

Like the Gauss and Codazzi-Mainardi equations, the compatibility conditions found in Theorem 3.1 reduces to only three scalar equations, since an antisymmetric matrix of order three has only three independent coefficients.

As expected, these three equations are equivalent to the Gauss and Codazzi-Mainardi equations; cf. Theorem 6.1.

A different set of necessary compatibility conditions, also related to a rotation field on a surface, has been proposed by Vallée \& Fortuné [30].

\section{SOME FUNDAMENTAL EXISTENCE AND UNIQUENESS THEOREMS FOR LINEAR DIFFERENTIAL SYSTEMS}

Our next objective is to show that the necessary compatibility conditions found in Theorem 3.1 are also sufficient for the existence of a surface if $\omega$ is simply-connected. Our proof will rely in an essential way on fundamental existence and uniqueness theorems for linear differential systems with little regularity (see Theorems 4.1 and 4.2 below) that are due to S. Mardare [21, Theorems 7 and 8]. Note that these existence theorems have been recently extended, again by S. Mardare [22, Theorems 3.6 and 6.5], to the $W_{\text {loc }}^{1, p}(\Omega)$-setting for any dimension $N \geq 2$, when $\Omega$ is a simply-connected open subset of $\mathbb{R}^{N}$ and $p>N$. Existence theorems in the $W_{\text {loc }}^{1, \infty}(\Omega)$-setting, again for any dimension $N \geq 2$, had been earlier obtained, also by S. Mardare [20, Theorem 3.1]. For smooth data, such existence results go back to Cartan [5] and Thomas [29].

The first theorem applies to Pfaff systems: 
Theorem 4.1. Let $\omega$ be a simply-connected open subset of $\mathbb{R}^{2}$, let $p>2$, and let $m \geq 1$ and $n \geq 1$ be integers. Let there be given matrix fields $\mathbf{A}_{\alpha} \in L_{\mathrm{loc}}^{p}\left(\omega ; \mathbb{M}^{n}\right)$ that satisfy

$$
\partial_{1} \mathbf{A}_{2}-\partial_{2} \mathbf{A}_{1}+\mathbf{A}_{1} \mathbf{A}_{2}-\mathbf{A}_{2} \mathbf{A}_{1}=\mathbf{0} \text { in } \mathscr{D}^{\prime}\left(\omega ; \mathbb{M}^{n}\right),
$$

and let a point $y_{0} \in \omega$ and a matrix $\mathbf{F}_{0} \in \mathbb{M}^{m \times n}$ be given. Then there exists one and only one matrix field $\mathbf{F} \in W_{\mathrm{loc}}^{1, p}\left(\omega ; \mathbb{M}^{m \times n}\right)$ that satisfies the Pfaff system

$$
\partial_{\alpha} \mathbf{F}=\mathbf{F} \mathbf{A}_{\alpha} \text { in } \mathscr{D}^{\prime}\left(\omega ; \mathbb{M}^{m \times n}\right), \quad \mathbf{F}\left(y_{0}\right)=\mathbf{F}_{0} .
$$

The second theorem is a Poincaré lemma with little regularity. Note that other Poincaré lemmas with little regularity, this time in the $H^{-1}(\omega)$-setting instead of the $L_{\text {loc }}^{p}(\omega)$-setting considered here, have been also recently established; see [8] and [2].

Theorem 4.2. Let $\omega$ be a simply-connected open subset of $\mathbb{R}^{2}$ and let $p \geq 1$. Let $h_{\alpha} \in$ $L_{\mathrm{loc}}^{p}(\omega)$ be functions that satisfy

$$
\partial_{1} h_{2}=\partial_{2} h_{1} \text { in } \mathscr{D}^{\prime}(\omega)
$$

Then there exists a function $p \in W_{\mathrm{loc}}^{1, p}(\omega)$, unique up to an additive constant, such that

$$
\partial_{\alpha} p=h_{\alpha} \text { in } L_{\mathrm{loc}}^{p}(\omega) .
$$

As shown by S. Mardare [22, Theorem 6.8], the existence and uniqueness result of Theorem 4.1 can be extended to one in the space $W^{1, p}\left(\omega ; \mathbb{M}^{m \times n}\right)$ (cf. Theorem 4.3). In order to state this extension, we recall the following definition: An open set $\omega \subset \mathbb{R}^{2}$ satisfies the uniform interior cone condition if there exists an open cone $\kappa$ such that, for every $y \in \omega$, there exists a cone $\kappa_{y}$ congruent to $\kappa$ (this means that $\kappa_{y}$ is obtained from $\kappa$ by a rigid motion), with vertex $y$, such that $\kappa_{y} \subset \omega$ (for details, see, e.g., Adams [1, Chapter 4]).

As a complement to Theorem 4.1, we then have:

Theorem 4.3. Let $\omega$ be a simply-connected bounded open subset of $\mathbb{R}^{2}$ that satisfies the uniform interior cone condition, let $p>2$, and let $m \geq 1$ and $n \geq 1$ be integers. Let there be given matrix fields $\mathbf{A}_{\alpha} \in L^{p}\left(\omega ; \mathbb{M}^{n}\right)$ that satisfy

$$
\partial_{1} \mathbf{A}_{2}-\partial_{2} \mathbf{A}_{1}+\mathbf{A}_{1} \mathbf{A}_{2}-\mathbf{A}_{2} \mathbf{A}_{1}=\mathbf{0} \text { in } \mathscr{D}^{\prime}\left(\omega ; \mathbb{M}^{n}\right),
$$

and let a point $y_{0} \in \omega$ and a matrix $\mathbf{F}_{0} \in \mathbb{M}^{m \times n}$ be given. Then there exists one and only one matrix field $\mathbf{F} \in W^{1, p}\left(\omega ; \mathbb{M}^{m \times n}\right)$ that satisfies the Pfaff system

$$
\partial_{\alpha} \mathbf{F}=\mathbf{F} \mathbf{A}_{\alpha} \text { in } \mathscr{D}^{\prime}\left(\omega ; \mathbb{M}^{m \times n}\right), \quad \mathbf{F}\left(y_{0}\right)=\mathbf{F}_{0} .
$$

Likewise, the existence result of Theorem 4.2 can be extended to the space $W^{1, p}(\omega)$ (the proof of this extension is analogous to that in [22, Theorem 6.8]):

Theorem 4.4. Let $\omega$ be a simply-connected bounded open subset of $\mathbb{R}^{2}$ that satisfies the uniform interior cone condition and let $p>1$. Let $h_{\alpha} \in L^{p}(\omega)$ be functions that satisfy

$$
\partial_{1} h_{2}=\partial_{2} h_{1} \text { in } \mathscr{D}^{\prime}(\omega)
$$

Then there exists a function $p \in W^{1, p}(\omega)$, unique up to an additive constant, such that

$$
\partial_{\alpha} p=h_{\alpha} \text { in } L^{p}(\omega) \text {. }
$$

We conclude this section with a uniqueness result that complements that of Theorem 4.1. 
Theorem 4.5. Let $\omega$ be a connected open subset of $\mathbb{R}^{2}$, let $p>2$, let $n \geq 1$ be an integer, let $\mathbf{B}_{\alpha}$ and $\mathbf{C}_{\alpha}$ be matrix fields in the space $L_{\mathrm{loc}}^{p}\left(\omega ; \mathbb{M}^{n}\right)$, and let a point $y_{0} \in \omega$ and $a$ matrix $\mathbf{F}_{0} \in \mathbb{M}^{n}$ be given. Then there exists at most one matrix field $\mathbf{F} \in W_{\mathrm{loc}}^{1, p}\left(\omega ; \mathbb{M}^{n}\right)$ that satisfies the Pfaff system

$$
\partial_{\alpha} \mathbf{F}=\mathbf{F B}_{\alpha}+\mathbf{C}_{\alpha} \mathbf{F} \text { in } \mathscr{D}^{\prime}\left(\omega ; \mathbb{M}^{n}\right), \quad \mathbf{F}\left(y_{0}\right)=\mathbf{F}_{0} .
$$

Proof. Let $m:=n^{2}$. If a matrix field $\mathbf{F}=\left(F_{i j}\right) \in W_{\text {loc }}^{1, p}\left(\omega ; \mathbb{M}^{n}\right)$ satisfies the above Pfaff system, then the matrix field $\tilde{\mathbf{F}} \in W_{\text {loc }}^{1, p}\left(\omega ; \mathbb{M}^{1 \times m}\right)$ defined by

$$
\tilde{\mathbf{F}}:=\left(F_{11} \cdots F_{1 n} F_{21} \cdots F_{2 n} \cdots F_{n 1} \cdots F_{n n}\right)
$$

satisfies a Pfaff system of the form

$$
\partial_{\alpha} \tilde{\mathbf{F}}=\tilde{\mathbf{F}} \tilde{\mathbf{A}}_{\alpha} \text { in } \mathscr{D}^{\prime}\left(\omega ; \mathbb{M}^{1 \times m}\right), \quad \tilde{\mathbf{F}}\left(y_{0}\right)=\tilde{\mathbf{F}}_{0},
$$

where the elements of the matrix fields $\tilde{\mathbf{A}}_{\alpha}: \omega \rightarrow \mathbb{M}^{m}$, which are linear combinations with constant coefficients of the elements of the matrix fields $\mathbf{B}_{\alpha}$ and $\mathbf{C}_{\alpha}$, thus belong to the space $L_{\mathrm{loc}}^{p}\left(\omega ; \mathbb{M}^{m}\right)$, and the matrix $\tilde{\mathbf{F}}_{0} \in \mathbb{M}^{1 \times m}$ is defined by

$$
\tilde{\mathbf{F}}_{0}:=\left(\begin{array}{llllllll}
F_{11}^{0} & \cdots & F_{1 n}^{0} & F_{21}^{0} & \cdots & F_{2 n}^{0} \cdots F_{n 1}^{0} \cdots F_{n n}^{0}
\end{array}\right) \text {, where } \mathbf{F}_{0}=\left(F_{i j}^{0}\right) .
$$

The conclusion then follows from the uniqueness result in S. Mardare [22, Theorem 4.2].

\section{SUFFICIENCY OF THE COMPATIBILITY CONDITIONS}

Under the assumption that the open set $\omega \subset \mathbb{R}^{2}$ is simply-connected, we now show that, if a field of positive-definite symmetric matrices $\left(a_{\alpha \beta}\right)$ and a field of symmetric matrices $\left(b_{\alpha \beta}\right)$, both defined on $\omega$, satisfy together the compatibility conditions that were found to be necessary in Theorem 3.1, then conversely, there exists an immersion $\theta: \omega \rightarrow \mathbb{R}^{3}$ such that $\left(a_{\alpha \beta}\right)$ and $\left(b_{\alpha \beta}\right)$ are the first and second fundamental forms of the surface $\boldsymbol{\theta}(\omega)$.

The assumption that $\omega$ is connected (recall that this assumption is contained in that of simple-connectedness) ensures that the solution in unique up to proper isometries of $\mathbb{R}^{3}$ (also known as rigid body motions in $\mathbb{R}^{3}$ ), i.e., any other solution $\tilde{\boldsymbol{\theta}}$ is such that $\tilde{\boldsymbol{\theta}}(y)=\mathbf{c}+$ $\mathbf{Q} \boldsymbol{\theta}(y)$ for all $y \in \omega$, for some vector $\mathbf{c} \in \mathbb{R}^{3}$ and some proper orthogonal matrix $\mathbf{Q} \in \mathbb{O}_{+}^{3}$. If otherwise $\omega$ is not connected, this uniqueness result holds over any connected component of $\omega$.

Theorem 5.1. Let $\omega$ be a simply-connected open subset of $\mathbb{R}^{2}$ and let $p>2$. Let there be given two matrix fields $\left(a_{\alpha \beta}\right) \in W_{\mathrm{loc}}^{1, p}\left(\omega ; \mathbb{S}_{>}^{2}\right)$ and $\left(b_{\alpha \beta}\right) \in L_{\mathrm{loc}}^{p}\left(\omega ; \mathbb{S}^{2}\right)$ that satisfy

$$
\partial_{1} \mathbf{A}_{2}-\partial_{2} \mathbf{A}_{1}+\mathbf{A}_{1} \mathbf{A}_{2}-\mathbf{A}_{2} \mathbf{A}_{1}=\mathbf{0} \text { in } \mathscr{D}^{\prime}\left(\omega ; \mathbb{A}^{3}\right)
$$

where the matrix fields $\mathbf{A}_{\alpha} \in L_{\mathrm{loc}}^{p}\left(\omega ; \mathbb{A}^{3}\right)$ are constructed from the matrix fields $\left(a_{\alpha \beta}\right)$ and $\left(b_{\alpha \beta}\right)$ by means of the following sequence of definitions:

$$
\begin{aligned}
& \Gamma_{\alpha \beta \tau}:=\frac{1}{2}\left(\partial_{\beta} a_{\alpha \tau}+\partial_{\alpha} a_{\beta \tau}-\partial_{\tau} a_{\alpha \beta}\right) \in L_{\mathrm{loc}}^{p}(\omega), \quad\left(a^{\sigma \tau}\right):=\left(a_{\alpha \beta}\right)^{-1} \in L_{\mathrm{loc}}^{p}\left(\omega ; \mathbb{S}_{>}^{2}\right), \\
& \Gamma_{\alpha \beta}^{\sigma}:=a^{\sigma \tau} \Gamma_{\alpha \beta \tau} \in L_{\mathrm{loc}}^{p}(\omega), \quad b_{\alpha}^{\sigma}:=a^{\beta \sigma} b_{\alpha \beta} \in L_{\mathrm{loc}}^{p}(\omega), \\
& \Gamma_{\alpha}:=\left(\begin{array}{llr}
\Gamma_{\alpha 1}^{1} & \Gamma_{\alpha 2}^{1} & -b_{\alpha}^{1} \\
\Gamma_{\alpha 1}^{2} & \Gamma_{\alpha 2}^{2} & -b_{\alpha}^{2} \\
b_{\alpha 1} & b_{\alpha 2} & 0
\end{array}\right) \in L_{\mathrm{loc}}^{p}\left(\omega ; \mathbb{M}^{3}\right), \quad \mathbf{C}:=\left(\begin{array}{rrr}
a_{11} & a_{12} & 0 \\
a_{21} & a_{22} & 0 \\
0 & 0 & 1
\end{array}\right) \in W_{\mathrm{loc}}^{1, p}\left(\omega ; \mathbb{S}_{>}^{3}\right), \\
& \mathbf{U}:=\mathbf{C}^{1 / 2} \in W_{\mathrm{loc}}^{1, p}\left(\omega ; \mathbb{S}_{>}^{3}\right), \quad \mathbf{A}_{\alpha}:=\left(\mathbf{U} \Gamma_{\alpha}-\partial_{\alpha} \mathbf{U}\right) \mathbf{U}^{-1} \in L_{\mathrm{loc}}^{p}\left(\omega ; \mathbb{A}^{3}\right) .
\end{aligned}
$$


Then there exists an immersion $\boldsymbol{\theta} \in W_{\mathrm{loc}}^{2, p}\left(\omega ; \mathbb{R}^{3}\right)$ such that

$$
a_{\alpha \beta}=\partial_{\alpha} \boldsymbol{\theta} \cdot \partial_{\beta} \theta \text { in } W_{\mathrm{loc}}^{1, p}(\omega) \quad \text { and } \quad b_{\alpha \beta}=\partial_{\alpha \beta} \boldsymbol{\theta} \cdot \frac{\partial_{1} \boldsymbol{\theta} \wedge \partial_{2} \boldsymbol{\theta}}{\left|\partial_{1} \boldsymbol{\theta} \wedge \partial_{2} \boldsymbol{\theta}\right|} \quad \text { in } L_{\mathrm{loc}}^{p}(\omega) .
$$

If any other immersion $\tilde{\boldsymbol{\theta}} \in W_{\mathrm{loc}}^{2, p}\left(\omega ; \mathbb{R}^{3}\right)$ satisfies the above relations (with $\tilde{\boldsymbol{\theta}}$ in lieu of $\boldsymbol{\theta}$ ), then there exists a vector $\mathbf{c} \in \mathbb{R}^{3}$ and a matrix $\mathbf{Q} \in \mathbb{O}_{+}^{3}$ such that

$$
\tilde{\boldsymbol{\theta}}(y)=\mathbf{c}+\mathbf{Q} \boldsymbol{\theta}(y) \text { for all } y \in \omega .
$$

Proof. For notational brevity, the function spaces are most often omitted in this proof.

(i) The matrix fields $\mathbf{A}_{\alpha}=\left(\mathbf{U} \boldsymbol{\Gamma}_{\alpha}-\partial_{\alpha} \mathbf{U}\right) \mathbf{U}^{-1}$ are antisymmetric. Since the matrices $\mathbf{U}(y)$ are symmetric and invertible at all $y \in \omega$, proving this property is the same as proving that the matrix fields

$$
\mathbf{U} \mathbf{A}_{\alpha} \mathbf{U}=\mathbf{C} \Gamma_{\alpha}-\mathbf{U} \partial_{\alpha} \mathbf{U}
$$

are themselves antisymmetric. A direct computation, based on the definition of the functions $\Gamma_{\alpha \beta \tau}, a^{\sigma \tau}, \Gamma_{\alpha \beta}^{\sigma}, b_{\alpha}^{\sigma}$ and of the matrix fields $\Gamma_{\alpha}$ and $\mathbf{C}$, shows that

$$
\mathbf{C} \Gamma_{\alpha}+\Gamma_{\alpha}^{T} \mathbf{C}=\partial_{\alpha} \mathbf{C}
$$

Consequently,

$$
\begin{aligned}
\mathbf{U} \mathbf{A}_{\alpha} \mathbf{U} & =\mathbf{C} \boldsymbol{\Gamma}_{\alpha}-\mathbf{U} \partial_{\alpha} \mathbf{U}=\frac{1}{2} \mathbf{C} \boldsymbol{\Gamma}_{\alpha}-\mathbf{U} \partial_{\alpha} \mathbf{U}+\frac{1}{2}\left(\partial_{\alpha} \mathbf{C}-\boldsymbol{\Gamma}_{\alpha}^{T} \mathbf{C}\right) \\
& =\frac{1}{2}\left(\mathbf{C} \boldsymbol{\Gamma}_{\alpha}-\boldsymbol{\Gamma}_{\alpha}^{T} \mathbf{C}\right)+\frac{1}{2}\left[\left(\partial_{\alpha} \mathbf{U}\right) \mathbf{U}+\mathbf{U} \partial_{\alpha} \mathbf{U}\right]-\mathbf{U} \partial_{\alpha} \mathbf{U} \\
& =\frac{1}{2}\left(\mathbf{C} \boldsymbol{\Gamma}_{\alpha}-\boldsymbol{\Gamma}_{\alpha}^{T} \mathbf{C}\right)+\frac{1}{2}\left[\left(\partial_{\alpha} \mathbf{U}\right) \mathbf{U}-\mathbf{U} \partial_{\alpha} \mathbf{U}\right],
\end{aligned}
$$

and thus the matrix fields $\mathbf{U} \mathbf{A}_{\alpha} \mathbf{U}$ are antisymmetric.

(ii) Let there be given a point $y_{0} \in \omega$ and a matrix $\mathbf{R}_{0} \in \mathbb{O}_{+}^{3}$. Then there exists one and only one matrix field $\mathbf{R} \in W_{\mathrm{loc}}^{1, p}\left(\omega ; \mathbb{O}_{+}^{3}\right)$ that satisfies

$$
\partial_{\alpha} \mathbf{R}=\mathbf{R} \mathbf{A}_{\alpha} \text { in } L_{\mathrm{loc}}^{p}\left(\omega ; \mathbb{M}^{3}\right), \quad \mathbf{R}\left(y_{0}\right)=\mathbf{R}_{0} .
$$

Since the matrix fields $\mathbf{A}_{\alpha}$ satisfy $\partial_{\alpha} \mathbf{A}_{\beta}-\partial_{\beta} \mathbf{A}_{\alpha}+\mathbf{A}_{\alpha} \mathbf{A}_{\beta}-\mathbf{A}_{\beta} \mathbf{A}_{\alpha}=\mathbf{0}$ in $\mathscr{D}^{\prime}\left(\omega ; \mathbb{A}^{3}\right)$, Theorem 4.1 provides the existence and uniqueness of a solution $\mathbf{R} \in W_{\text {loc }}^{1, p}\left(\omega ; \mathbb{M}^{3}\right)$. In order to show that this matrix field $\mathbf{R}$ is proper orthogonal, we note that the matrix field $\mathbf{R}^{T} \mathbf{R} \in W_{\text {loc }}^{1, p}\left(\omega, \mathbb{M}^{3}\right)$ satisfies the differential system

$$
\begin{aligned}
\partial_{\alpha}\left(\mathbf{R}^{T} \mathbf{R}\right) & =\left(\partial_{\alpha} \mathbf{R}\right)^{T} \mathbf{R}+\mathbf{R}^{T} \partial_{\alpha} \mathbf{R}=\mathbf{A}_{\alpha}^{T}\left(\mathbf{R}^{T} \mathbf{R}\right)+\left(\mathbf{R}^{T} \mathbf{R}\right) \mathbf{A}_{\alpha} \text { in } L_{\mathrm{loc}}^{p}\left(\omega ; \mathbb{M}^{3}\right), \\
\left(\mathbf{R}^{T} \mathbf{R}\right)\left(y_{0}\right) & =\mathbf{I} .
\end{aligned}
$$

Because the matrix fields $\mathbf{A}_{\alpha}$ are antisymmetric by part (i), $\mathbf{R}^{T} \mathbf{R}=\mathbf{I}$ is a solution to this system, and it is its unique solution by Theorem 4.5. Hence the matrix field $\mathbf{R}$ is orthogonal. In order to show that it is proper orthogonal, we note that $\mathbf{R} \in W_{\text {loc }}^{1, p}\left(\omega ; \mathbb{M}^{3}\right) \subset \mathscr{C}^{0}\left(\omega ; \mathbb{M}^{3}\right)$. Hence $\operatorname{det} \mathbf{R}(y)=1$ for all $y \in \omega$ since $\operatorname{det} \mathbf{R}\left(y_{0}\right)=\operatorname{det} \mathbf{R}_{0}=1$ and $\omega$ is connected.

(iii) The matrix field $\mathbf{R} \in W_{\mathrm{loc}}^{1, p}\left(\omega ; \mathbb{O}_{+}^{3}\right)$ being that determined in (ii), there exists an immersion $\boldsymbol{\theta} \in W_{\mathrm{loc}}^{2, p}\left(\omega, \mathbb{R}^{3}\right)$ that satisfies

$$
\partial_{\alpha} \boldsymbol{\theta}=\mathbf{R} \mathbf{u}_{\alpha} \text { in } W_{\mathrm{loc}}^{1, p}\left(\omega ; \mathbb{R}^{3}\right),
$$

where $\mathbf{u}_{\alpha}:=[\mathbf{U}]_{\alpha} \in W_{\mathrm{loc}}^{1, p}\left(\omega ; \mathbb{R}^{3}\right)$. 
Resorting this time to Theorem 4.2, we conclude that this system has a solution $\boldsymbol{\theta} \in$ $W_{\text {loc }}^{1, p}\left(\omega ; \mathbb{R}^{3}\right)$ if the compatibility relations

$$
\partial_{\beta}\left(\mathbf{R} \mathbf{u}_{\alpha}\right)=\partial_{\alpha}\left(\mathbf{R} \mathbf{u}_{\beta}\right) \text { in } L_{\text {loc }}^{p}\left(\omega ; \mathbb{R}^{3}\right)
$$

are satisfied. In view of the relations $\partial_{\alpha} \mathbf{R}=\mathbf{R} \mathbf{A}_{\alpha}$ (cf. part (ii)), we thus need to check that

$$
\mathbf{R} \mathbf{A}_{\beta} \mathbf{u}_{\alpha}+\mathbf{R} \partial_{\beta} \mathbf{u}_{\alpha}=\mathbf{R} \mathbf{A}_{\alpha} \mathbf{u}_{\beta}+\mathbf{R} \partial_{\alpha} \mathbf{u}_{\beta} \text { with } \mathbf{A}_{\alpha}=\left(\mathbf{U} \Gamma_{\alpha}-\partial_{\alpha} \mathbf{U}\right) \mathbf{U}^{-1},
$$

or equivalently (since the matrices $\mathbf{R}(y)$ are invertible at all points $y \in \omega$ ), that

$$
\left(\mathbf{U} \Gamma_{\beta}-\partial_{\beta} \mathbf{U}\right) \mathbf{U}^{-1} \mathbf{u}_{\alpha}+\partial_{\beta} \mathbf{u}_{\alpha}=\left(\mathbf{U} \Gamma_{\alpha}-\partial_{\alpha} \mathbf{U}\right) \mathbf{U}^{-1} \mathbf{u}_{\beta}+\partial_{\alpha} \mathbf{u}_{\beta}
$$

But a straightforward computation shows that this relation reduces in fact to the relation

$$
\left[\mathbf{U} \Gamma_{1}\right]_{2}=\left[\mathbf{U} \Gamma_{2}\right]_{1} \text {, with }\left[\mathbf{U} \Gamma_{1}\right]_{2}=\mathbf{U}\left(\begin{array}{c}
\Gamma_{12}^{1} \\
\Gamma_{12}^{2} \\
b_{12}
\end{array}\right) \text { and }\left[\mathbf{U} \Gamma_{2}\right]_{1}=\mathbf{U}\left(\begin{array}{c}
\Gamma_{21}^{1} \\
\Gamma_{21}^{2} \\
b_{21}
\end{array}\right) \text {. }
$$

Hence the assertion follows from the symmetries $\Gamma_{\alpha \beta}^{\sigma}=\Gamma_{\beta \alpha}^{\sigma}$ and $b_{\alpha \beta}=b_{\beta \alpha}$.

The existence of a vector field $\boldsymbol{\theta} \in W_{\mathrm{loc}}^{1, p}\left(\omega ; \mathbb{R}^{3}\right)$ that satisfies $\partial_{\alpha} \boldsymbol{\theta}=\mathbf{R} \mathbf{u}_{\alpha}$ in $L_{\mathrm{loc}}^{p}\left(\omega ; \mathbb{R}^{3}\right)$ is thus established. Since the fields $\mathbf{R}$ and $\mathbf{u}_{\alpha}$ are respectively in the spaces $W_{\text {loc }}^{1, p}\left(\omega ; \mathbb{O}_{+}^{3}\right)$ and $W_{\text {loc }}^{1, p}\left(\omega ; \mathbb{R}^{3}\right)$, it follows that $\boldsymbol{\theta} \in W_{\mathrm{loc}}^{2, p}\left(\omega ; \mathbb{R}^{3}\right)$. Since the vectors $\mathbf{u}_{\alpha}(y)$ are linearly independent and the matrix $\mathbf{R}(y)$ is proper orthogonal at all points $y \in \omega$, it further follows that $\boldsymbol{\theta}$ is an immersion.

(iv) The given matrix field $\left(a_{\alpha \beta}\right) \in W_{\mathrm{loc}}^{1, p}\left(\omega ; \mathbb{S}_{>}^{2}\right)$ is the first fundamental form of the surface $\boldsymbol{\theta}(\omega)$. Define the matrix and vector fields

$$
\mathbf{F}:=\mathbf{R} \mathbf{U} \in W_{\mathrm{loc}}^{1, p}\left(\omega ; \mathbb{M}^{3}\right) \quad \text { and } \quad \mathbf{f}_{j}:=[\mathbf{F}]_{j} \in W_{\mathrm{loc}}^{1, p}\left(\omega ; \mathbb{R}^{3}\right), 1 \leq j \leq 3,
$$

where $\mathbf{R} \in W_{\text {loc }}^{1, p}\left(\omega ; \mathbb{O}_{+}^{3}\right)$ is the matrix field found in (ii), $\mathbf{U}=\mathbf{C}^{1 / 2} \in W_{\text {loc }}^{1, p}\left(\omega ; \mathbb{S}_{>}^{3}\right)$, and the matrix field $\mathbf{C}$ is defined in terms of the functions $a_{\alpha \beta}$ as in the statement of the theorem. Then the relation $\mathbf{F}^{T} \mathbf{F}=\mathbf{C}$ and the specific form of the matrix field $\mathbf{C}$ imply that $\mathbf{f}_{\alpha}^{T} \mathbf{f}_{\beta}=a_{\alpha \beta}$ on the one hand, and the relations $\mathbf{F}=\mathbf{R} \mathbf{U}$ and $\partial_{\alpha} \boldsymbol{\theta}=\mathbf{R} \mathbf{u}_{\alpha}$ (cf. part (iii)) imply that $\mathbf{f}_{\alpha}=\partial_{\alpha} \boldsymbol{\theta}$ on the other. Hence

$$
\partial_{\alpha} \boldsymbol{\theta} \cdot \partial_{\beta} \boldsymbol{\theta}=a_{\alpha \beta} .
$$

(v) The given matrix field $\left(b_{\alpha \beta}\right) \in L_{\mathrm{loc}}^{p}\left(\omega ; \mathbb{S}^{2}\right)$ is the second fundamental form of the surface $\boldsymbol{\theta}(\omega)$. The relation $\mathbf{F}^{T} \mathbf{F}=\mathbf{C}$ and the specific form of the matrix field $\mathbf{C}$ imply that $\mathbf{f}_{i}^{T} \mathbf{f}_{3}=\delta_{i 3}$. Hence either $\mathbf{f}_{3}=\frac{\mathbf{f}_{1} \wedge \mathbf{f}_{2}}{\left|\mathbf{f}_{1} \wedge \mathbf{f}_{2}\right|}$ or $\mathbf{f}_{3}=-\frac{\mathbf{f}_{1} \wedge \mathbf{f}_{2}}{\left|\mathbf{f}_{1} \wedge \mathbf{f}_{2}\right|}$. But $\operatorname{det} \mathbf{F}(y)=\operatorname{det} \mathbf{R}(y) \operatorname{det} \mathbf{U}(y)>$ 0 for all $y \in \omega$. Hence

$$
\mathbf{f}_{3}=\frac{\mathbf{f}_{1} \wedge \mathbf{f}_{2}}{\left|\mathbf{f}_{1} \wedge \mathbf{f}_{2}\right|}
$$

on the one hand. On the other hand,

$$
\begin{aligned}
\partial_{\alpha \beta} \boldsymbol{\theta} & =\partial_{\beta}\left(\mathbf{R} \mathbf{u}_{\alpha}\right)=\left(\partial_{\beta} \mathbf{R}\right) \mathbf{u}_{\alpha}+\mathbf{R} \partial_{\beta} \mathbf{u}_{\alpha}=\mathbf{R} \mathbf{A}_{\beta} \mathbf{u}_{\alpha}+\mathbf{R} \partial_{\beta} \mathbf{u}_{\alpha} \\
& =\mathbf{R}\left(\mathbf{A}_{\beta} \mathbf{u}_{\alpha}+\partial_{\beta} \mathbf{u}_{\alpha}\right)=\mathbf{R}\left[\mathbf{A}_{\beta} \mathbf{U}+\partial_{\beta} \mathbf{U}\right]_{\alpha}=\mathbf{R}\left[\mathbf{U} \boldsymbol{\Gamma}_{\beta}\right]_{\alpha} \\
& =\left[\mathbf{R} \mathbf{U} \boldsymbol{\Gamma}_{\beta}\right]_{\alpha}=\left[\mathbf{F} \boldsymbol{\Gamma}_{\beta}\right]_{\alpha} .
\end{aligned}
$$


Consequently,

$$
\partial_{\alpha \beta} \boldsymbol{\theta} \cdot \mathbf{f}_{3}=\left(\partial_{\alpha \beta} \boldsymbol{\theta}\right)^{T} \mathbf{f}_{3}=\left[\boldsymbol{\Gamma}_{\beta}\right]_{\alpha}^{T} \mathbf{F}^{T} \mathbf{f}_{3}=\left(\Gamma_{\beta \alpha}^{1} \Gamma_{\beta \alpha}^{2} b_{\beta \alpha}\right)\left(\begin{array}{l}
0 \\
0 \\
1
\end{array}\right)=b_{\beta \alpha} .
$$

(vi) The uniqueness of the immersion $\boldsymbol{\theta} \in W_{\text {loc }}^{1, p}\left(\omega ; \mathbb{R}^{3}\right)$ up to proper isometries of $\mathbb{R}^{3}$ follows from the rigidity theorem with little regularity established in [13, Theorem 3].

An inspection of the above proof immediately leads to an existence result in spaces of continuously differentiable functions:

Theorem 5.2. Assume in Theorem 5.1 that the matrix fields $\left(a_{\alpha \beta}\right)$ and $\left(b_{\alpha \beta}\right)$ respectively belong to the spaces $\mathscr{C}^{m+1}\left(\omega ; \mathbb{S}_{>}^{2}\right)$ and $\mathscr{C}^{m}\left(\omega ; \mathbb{S}^{2}\right)$ for some integer $m \geq 0$, all the other assumptions and definitions of Theorem 5.1 holding verbatim. Then the immersion $\boldsymbol{\theta}$ found in Theorem 5.1 belongs to the space $\mathscr{C}^{m+2}\left(\omega ; \mathbb{R}^{3}\right)$.

Under an additional assumption on the set $\omega$, a similar existence result holds in the space $W^{2, p}\left(\omega ; \mathbb{R}^{3}\right)$.

Theorem 5.3. Assume in Theorem 5.1 that $\omega$ is bounded and satisfies the uniform interior cone condition and that the matrix fields $\left(a_{\alpha \beta}\right)$ and $\left(b_{\alpha \beta}\right)$ respectively belong to the spaces $W^{1, p}\left(\omega ; \mathbb{S}_{>}^{2}\right)$ and $L^{p}\left(\omega ; \mathbb{S}^{2}\right)$, all the other assumptions and definitions of Theorem 5.1 holding verbatim. Then the immersion $\boldsymbol{\theta}$ found in Theorem 5.1 belongs to the space $W^{2, p}\left(\omega, \mathbb{R}^{3}\right)$.

Proof. The proof is analogous to that of Theorem 5.1, save that the existence results of Theorems 4.1 and 4.2 are now replaced by those of Theorems 4.3 and 4.4.

\section{EQUIVALENCE BETWEEN THE NEW COMPATIBILITY CONDITIONS AND THE GAUSS AND CODAZZI-MAINARDI EQUATIONS.}

To conclude our analysis, we establish the equivalence between the compatibility conditions of Theorems 3.1 or 5.1 and the Gauss and Codazzi-Mainardi equations.

Theorem 6.1. Let $\omega$ be an open subset of $\mathbb{R}^{2}$. Then two matrix fields $\left(a_{\alpha \beta}\right) \in W_{\mathrm{loc}}^{1, p}\left(\omega ; \mathbb{S}_{>}^{2}\right)$ and $\left(b_{\alpha \beta}\right) \in L_{\mathrm{loc}}^{p}\left(\omega ; \mathbb{S}^{2}\right)$ satisfy the matrix equation

$$
\partial_{1} \mathbf{A}_{2}-\partial_{2} \mathbf{A}_{1}+\mathbf{A}_{1} \mathbf{A}_{2}-\mathbf{A}_{2} \mathbf{A}_{1}=\mathbf{0} \text { in } \mathscr{D}^{\prime}\left(\omega ; \mathbb{A}^{3}\right),
$$

where the matrix fields $\mathbf{A}_{\alpha} \in L_{\mathrm{loc}}^{p}\left(\omega ; \mathbb{A}^{3}\right)$ are constructed from the matrix fields $\left(a_{\alpha \beta}\right)$ and $\left(b_{\alpha \beta}\right)$ as in Theorems 3.1 or 5.1, if and only if they satisfy the Gauss and Codazzi-Mainardi equations in the space $\mathscr{D}^{\prime}(\omega)$.

Proof. Since the equivalence between the two sets of compatibility conditions is a "local" property, we may assume without loss of generality that $\omega$ is simply-connected. Assume that two matrix fields $\left(a_{\alpha \beta}\right) \in W_{\mathrm{loc}}^{1, p}\left(\omega ; \mathbb{S}_{>}^{2}\right)$ and $\left(b_{\alpha \beta}\right) \in L_{\mathrm{loc}}^{p}\left(\omega ; \mathbb{S}^{2}\right)$ satisfy the above matrix equation in $\mathscr{D}^{\prime}\left(\omega ; \mathbb{A}^{3}\right)$. Then, by Theorem 5.1 , there exists an immersion $\boldsymbol{\theta} \in W_{\mathrm{loc}}^{2, p}\left(\omega ; \mathbb{R}^{3}\right)$ such that $\left(a_{\alpha \beta}\right)$ and $\left(b_{\alpha \beta}\right)$ are the first and second fundamental forms of the surface $\boldsymbol{\theta}(\omega)$. Hence they necessarily satisfy the Gauss and Codazzi-Mainardi equations in $\mathscr{D}^{\prime}(\omega)$. 
Assume conversely that two matrix fields $\left(a_{\alpha \beta}\right) \in W_{\mathrm{loc}}^{1, p}\left(\omega ; \mathbb{S}_{>}^{2}\right)$ and $\left(b_{\alpha \beta}\right) \in L_{\mathrm{loc}}^{p}\left(\omega ; \mathbb{S}^{2}\right)$ satisfy the Gauss and Codazzi-Mainardi equations in $\mathscr{D}^{\prime}(\omega)$. Then, thanks to the fundamental theorem of surface theory "with little regularity" of S. Mardare [21], there exists an immersion $\boldsymbol{\theta} \in W_{\mathrm{loc}}^{2, p}\left(\omega ; \mathbb{R}^{3}\right)$ such that $\left(a_{\alpha \beta}\right)$ and $\left(b_{\alpha \beta}\right)$ are the two fundamental forms of the surface $\theta(\omega)$. Hence the two matrix fields necessarily satisfy the above matrix equation in $\mathscr{D}^{\prime}\left(\omega ; \mathbb{A}^{3}\right)$ by Theorem 3.1.

The two sets of compatibility conditions are therefore equivalent.

Acknowledgement. The work described in this paper was substantially supported by a grant from the Research Grants Council of the Hong Kong Special Administrative Region, China [Project No.9040966, City U 100604].

\section{REFERENCES}

[1] R. A. Adams: Sobolev Spaces. Academic Press, New York, 1975

[2] C. Amrouche, P. G. Ciarlet, L. Gratie, S. Kesavan: On the characterizations of matrix fields as linearized strain tensor fields. J. Math. Pures Appl. 86, 116-132 (2006)

[3] S. S. AnTman: Ordinary differential equations of nonlinear elasticity I: Foundations of the theories of nonlinearly elastic rods and shells. Arch. Rational Mech. Anal. 61, 307-351 (1976)

[4] Y. BAŞAR: A consistent theory of geometrically non-linear shells with an independent rotation vector. Internat. J. Solids Structures 23, 1401-1445 (1987)

[5] E. Cartan: La Géométrie des Espaces de Riemann, Mémorial des Sciences Mathématiques, Fasc. 9. Gauthier-Villars, Paris, 1925

[6] P. G. Ciarlet: Mathematical Elasticity, Volume I: Three-Dimensional Elasticity. North-Holland, Amsterdam, 1988

[7] P. G. Ciarlet: An Introduction to Differential Geometry with Applications to Elasticity. Springer, Dordrecht, 2005

[8] P. G. Ciarlet, P. Ciarlet JR.: Another approach to linearized elasticity and a new proof of Korn's inequality. Math. Models Methods Appl. Sci. 15, 259-271 (2005)

[9] P. G. Ciarlet, L. Gratie, C. Mardare: A nonlinear Korn inequality on a surface. J. Math. Pures Appl. 85, 2-16 (2006)

[10] P. G. Ciarlet, L. Gratie, C. Mardare: New compatibility conditions for the fundamental theorem of surface theory. C.R. Acad. Sci. Paris, Ser. I 345, 273-278 (2007)

[11] P. G. Ciarlet, L. Gratie, O. Iosifescu, C. Mardare, C. Vallée: Another approach to the fundamental theorem of Riemannian geometry in $\mathbb{R}^{3}$, by way of rotation fields. J. Math. Pures Appl. 87, 237-252 (2007)

[12] P. G. Ciarlet, F. LARSONnEuR: On the recovery of a surface with prescribed first and second fundamental forms. J. Math. Pures Appl. 81, 167-185 (2001)

[13] P. G. Ciarlet, C. Mardare: On rigid and infinitesimal rigid displacements in shell theory. J. Math. Pures Appl. 83, 1-15 (2004)

[14] P. G. Ciarlet, C. Mardare: Continuity of a deformation in $H^{1}$ as a function of its Cauchy-Green tensor in $L^{1}$. Nonlinear Sci. 14, 415-427 (2004)

[15] P. G. Ciarlet, C. Mardare: Recovery of a surface with boundary and its continuity as a function of its fundamental forms. Analysis and Applications 3, 99-117 (2005)

[16] A. Galka, J. J. Telega: The complementary energy principle as a dual problem for a specific model of geometrically nonlinear elastic shells with an independent rotation vector: general results. European J. Mech. A Solids 11, 245-270 (1992)

[17] M. E. GuRTin: An Introduction to Continuum Mechanics. Academic Press, New York, 1981.

[18] P. HARTMAn, A. WinTnER: On the embedding problem in differential geometry. Amer. J. Math. 72, 553564 (1950)

[19] M. JANET: Sur la possibilité de plonger un espace riemannien donné dans un espace euclidien. Ann. Soc. Polon. Math. 5, 38-43 (1926)

[20] S. MARDARE: The fundamental theorem of surface theory for surfaces with little regularity. J. Elasticity 73, 251-290 (2003) 
[21] S. Mardare: On Pfaff systems with $L^{p}$ coefficients and their applications in differential geometry. J. Math. Pures Appl. 84, 1659-1692 (2005)

[22] S. MARDARE: On systems of first order linear partial differential equations with $L^{p}$ coefficients. Advances in Differential Equations 12, 301-360 (2007)

[23] W. PietraszKiewicZ: Finite Rotations and Lagrangean Description in the Non-Linear Theory of Shells. Polish Scientific Publishers, Warszawa, 1979

[24] W. PietraszKiewicz: On using rotations as primary variables in the nonlinear theory of thin irregular shells, in D. Durban et al. (Editors), Advances in the Mechanics of Plates and Shells, pp. 245-258, Kluwer, 2001

[25] W. Pietraszkiewicz, J. Badur: Finite rotations in the description of continuum deformation. Internat. J. Engrg. Sci. 21, 1097-1115 (1983)

[26] R. T. SHIELD: The rotation associated with large strains. SIAM J. Appl. Math. 25, 483-491 (1973)

[27] J. G. Simmonds, D. A. DANiELSON: Nonlinear shell theory with finite rotation and stress function vectors. J. Appl. Mech. 39, 1085-1090 (1972)

[28] J. C. Simo, D. D. Fox: On stress resultant geometrically exact shell model, Part 1: Formulation and optimal parametrization, Computer Methods Appl. Mech. Engrg. 72, 267-304 (1989)

[29] T. Y. Thomas: Systems of total differential equations defined over simply-connected domains. Annals of Mathematics 35, 730-734 (1934)

[30] C. Vallée, D. Fortuné: Compatibility equations in shell theory. Internat. J. Engrg. Sci. 34, 495-499 (1996)

[31] R. VALID: The principle of complementary energy in nonlinear shell theory. C.R. Acad. Sci. Paris B289, 293-296 (1979)

Philippe G. Ciarlet: Department of Mathematics, City University of Hong Kong, 83 Tat Chee Avenue, Kowloon, Hong Kong, EmaIl:MapgC@ Cityu.edu.hK

Liliana Gratie: Liu Bie Ju Centre for Mathematical Sciences, City University of Hong Kong, 83 Tat Chee Avenue, Kowloon, Hong Kong, EmaIl:mCgratie@ Cityu.edu.hK

Cristinel Mardare: Laboratoire Jacques - Louis Lions, Université Pierre et Marie Curie, 4 Place Jussieu, 75005 PARIS, France, EMAIL:MARDARE@ ANN.JUSSIEU.FR 